BASIC RESEARCH

\title{
Transarterial aortic valve replacement with a self expanding stent in pigs
}

\author{
M Ferrari, H R Figulla, M Schlosser, I Tenner, I Frerichs, C Damm, V Guyenot, G S Werner, \\ G Hellige
}

Heart 2004;90:1326-1331. doi: 10.1136/hrt.2003.028951

See end of article for authors' affiliations .....................

Correspondence to: Dr Markus Ferrari, Clinic of Internal Medicine I, Friedrich-Schiller University, Erlanger Allee 101, D-07740 Jena, Germany; markus.ferrari@ med.uni-jena.de

Accepted 24 March 2004
Objectives: To evaluate the feasibility of percutaneous aortic valve replacement without cardiac arrest in animal experiments.

Methods: A self expanding nitinol stent, containing pulmonary valves from pigs in its proximal part, was implanted in six pigs $(94-118 \mathrm{~kg}$ ) by means of a 25 French catheter through the left subclavian artery under guidance of fluoroscopy and transoesophageal echocardiography. During stent deployment the original aortic valve was pushed against the aortic wall by the self expanding force of the stent while the new valve was expanded.

Results: It was possible to replace the aortic valve in the beating heart in four pigs $(67 \%)$ with no complication or relevant drop in blood pressure. The procedure failed in two pigs (33\%) due to dysfunction of the catheter device in one case and to problems with correct positioning in the left ventricular outflow tract in the other. After successful stent valve implantation, dopamine was infused in doses of $5 \mu \mathrm{g} / \mathrm{kg} / \mathrm{min}$, $10 \mu \mathrm{g} / \mathrm{kg} / \mathrm{min}$, and $15 \mu \mathrm{g} / \mathrm{kg} / \mathrm{min}$. Cardiac output increased from 4.4 to $8.8 \mathrm{l} / \mathrm{min}$ and the mean arterial pressure rose from 79 to $105 \mathrm{~mm} \mathrm{Hg}$. The maximum peak to peak pressure gradient across the valve carrying stent reached a maximum of $8 \mathrm{~mm} \mathrm{Hg}$ under dopamine infusion. All pigs were killed six hours after transvascular aortic valve replacement. The chest was opened, and the left ventricle and the ascending aorta were carefully inspected. There were no signs of malfunction of the implant, of damage of the aortic vessel wall, or of obstruction of the coronary ostia.

Conclusions: Percutaneous aortic valve replacement with a self expanding nitinol stent in the beating heart is possible. The device was safe under pharmacological stress test. After successful chronic animal experiments, this concept may become a feasible option for treating patients with relevant aortic valve disease but where open heart surgery would be risky.
$\mathrm{P}$ atients with a stenosis of the aortic valve do not show any symptoms for several years. But when they become symptomatic, their prognosis is limited if the valve is not replaced. ${ }^{1}$ The prevalence of severe calcification of the aortic valve is $13 \%$ in patients 75 years and older. ${ }^{2}$ The number of elderly patients with relevant co-morbidity and symptomatic aortic valve disease is increasing. These patients are often high risk candidates for open heart surgery with a heart-lung machine. ${ }^{3}$ Recently, a one month mortality rate of nearly $24 \%$ was reported among patients 90 years and older after open heart valve surgery. ${ }^{4}$ Thus, there is need for a less invasive technique of aortic valve replacement. Several groups have been working on this field during the past decade..$^{5-8}$

Bonhoeffer and colleagues ${ }^{9}$ were the first to implant a valve percutaneously with a transvenous approach in a human. They sutured a bovine jugular vein valve within a balloon expandable stent for replacing the pulmonary valve. Cribier and colleagues ${ }^{10}$ also used a biological valve mounted in a balloon expandable stent, which was implanted in a transseptal approach. These first human cases started a new era of interventional cardiology. However, no data about pharmacological stress tests and no chronic animal experiments are available.

All approaches to percutaneous valve replacement to date have used a balloon expandable stent that may loosen as a result of continuous mechanical movement in the left ventricular outlet tract and retraction of the surrounding tissue. Thus, we developed a concept of percutaneous aortic valve replacement with a self expanding stent. The advantages of this stent are its lower crossing profile, the distribution of mechanical forces up to the ascending aorta, and its ability adapt geometrically if the conformation and the structure of the surrounding tissue change over time.

It was the aim of this study to evaluate the feasibility of percutaneous aortic valve replacement without cardiac arrest. Furthermore, we wanted to test the haemodynamic performance of a percutaneously implanted stent valve device during increased heart rate or high blood pressure.

\section{METHODS \\ Design of the stent valve}

The self expanding stent is made of the memory metal nitinol. The nitinol stent is flexible and foldable at room temperature. Above $25^{\circ} \mathrm{C}$, it becomes rigid and rapidly expands to its defined diameter of $25 \mathrm{~mm}$. The design of the $6 \mathrm{~cm}$ long stent was adapted to the left ventricular outflow tract. The thickness of the struts is $160 \mu \mathrm{m}$. The stent is anchored by barbed hooks in the ascending aorta. These hooks and the self expanding force of the stent prevent retrograde dislocation of the stent valve device into the left ventricle after its complete deployment out of the catheter (fig 1). The self expanding stent rapidly expands in the left ventricular outlet tract after warming above $25^{\circ} \mathrm{C}$. The native valve is pushed against the aortic wall by the expanding force of the stent. At the same time, the new valve unfolds in the aortic position taking over the function of the native valve.

\section{Preparation of the biological valve prosthesis}

Pig hearts were obtained from the local slaughterhouse. Pulmonary valves were carefully prepared. The valves were 

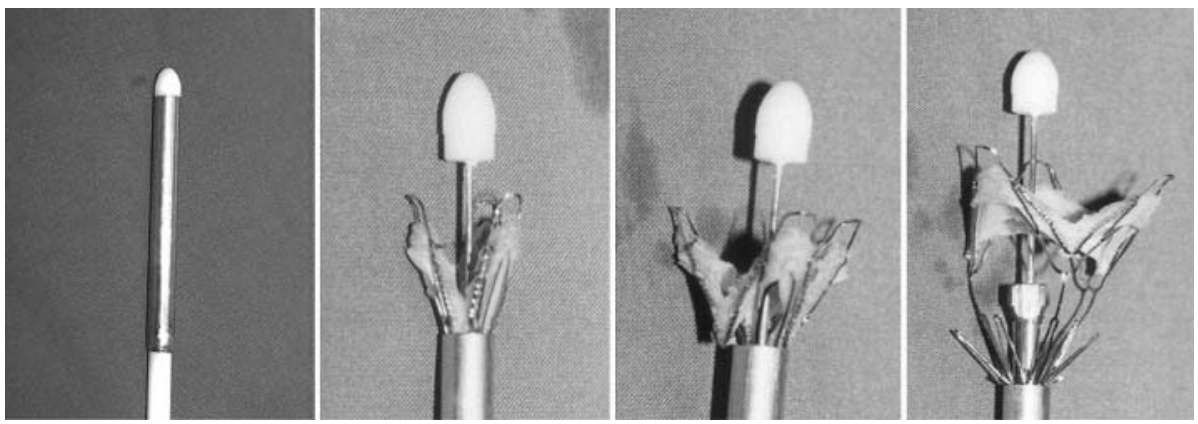

Figure 1 Deployment of the self expanding stent valve out of the tip of the catheter in sequential steps: after contact with blood the nitinol stent reaches its transformation temperature leading to an expansion of the proximal segment that carried the valve. After optimal positioning the distal part of the stent is released out of the catheter stabilising and anchoring the stent in the ascending aorta.

trimmed under a magnifying glass to remove unnecessary material and to reduce the thickness as much as possible. After low pressure fixation with $0.25 \%$ glutaraldehyde, the valves were mounted in the self expanding stent by sutures along the commissures with 5-0 polypropylene thread. The expanded valves had diameters between 20 and $24 \mathrm{~mm}$. The stent valves were stored in the refrigerator at $4^{\circ} \mathrm{C}$ until implantation.

\section{Construction of the implantation catheter}

The catheter had an outer diameter of 25 French. The flexible catheter allowed cold saline to be flushed continuously through its lumen to keep the temperature of the stent below $25^{\circ} \mathrm{C}$ during the implantation procedure. The catheter had an inner channel for a stiff guidewire. The stent was inserted in the tip of the catheter. The stent was stabilised in the catheter by an inner shaft with three circumferential ridges. This technology allowed exactly controlled release of the stent. It also allowed retraction of the stent back into the catheter if the stent was not completely released out of the catheter. Moving the catheter backward and forward and rotating the inner shaft allowed for optimal positioning of the stent valve before definitive deployment.

\section{Implantation procedure}

In six pigs (94-118 kg) we induced general anaesthesia with intravenous thiopental $(10 \mathrm{mg} / \mathrm{kg})$ after intramuscular injection of midazolam $(0.25 \mathrm{mg} / \mathrm{kg})$. We used halothane to maintain narcosis. Arterial blood gas was sampled often to optimise mechanical ventilation. All animals were treated according to the European regulations for animal experiments. ${ }^{11}$ At the end of the experiments, the animals were killed by intracoronary injection of $20 \mathrm{mmol}$ potassium chloride.

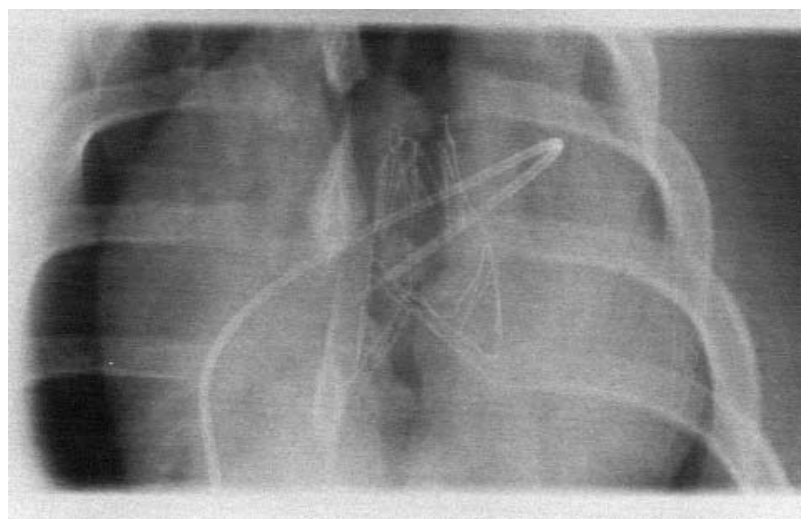

Figure 2 Chest radiograph showing the stent valve after implantation through the subclavian artery. A Swan-Ganz catheter was inserted in the pulmonary artery from the femoral vein.
Standard catheterisation was done with a Swan-Ganz catheter and a 5 French pigtail catheter from the right femoral artery to record pressure and for transaortic pullback. Cardiac output ( $\mathrm{CO}$ ) was measured by the thermodilution technique. Three consecutive measurements were averaged for further analysis. A transoesophageal echocardiography (TOE) probe was inserted to monitor the aortic valve. The size of the aortic ring was estimated by TOE before the implantation procedure was started. We measured the flow velocity in the aortic valve with continuous wave Doppler. All values are presented as mean (SD) of measurements in four pigs.

After establishing baseline parameters we implanted the stent valve through an approach from the left subclavian artery. We selected the size of our stent valve according to the TOE measurements. By fluoroscopic guidance a 0.035 inch guidewire was advanced in the left ventricle. The 25 French catheter was inserted in the angle between the left subclavian artery and left carotid artery over the guidewire. Under continuous flushing with cold saline, the catheter was advanced to the left ventricle. The outer part of the catheter was slowly retracted and the stent valve was partly deployed. By TOE and fluoroscopic guidance the position of the prosthetic valve was optimised (fig 2) and the stent was completely released out of the catheter and anchored in the ascending aorta.

After implantation of the stent valve the catheter was carefully retracted and a tobacco bag suture secured the insertion site. After catheterisation a bolus of heparin (100 IU/kg) was given and an additional $50 \mathrm{IU} / \mathrm{kg}$ of heparin was administered every two hours during the experiment.

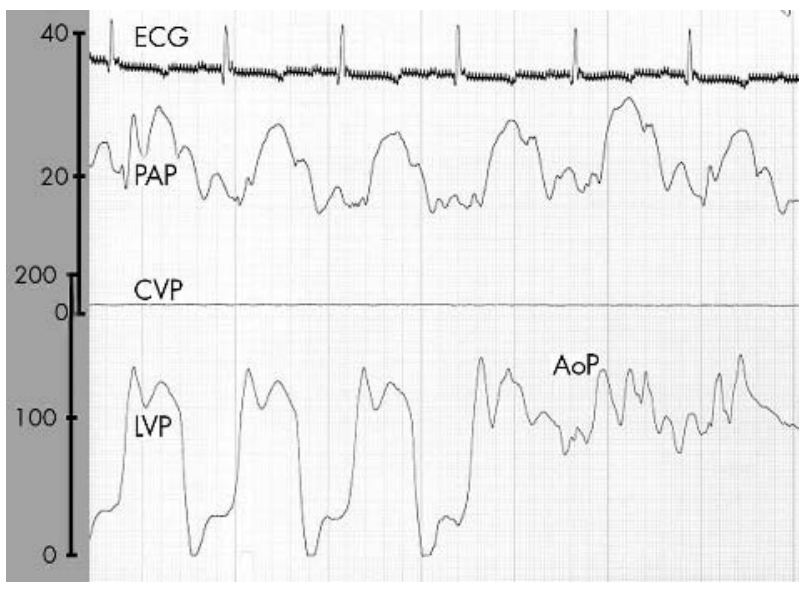

Figure 3 Continuous recordings of ECG, pulmonary artery pressure, (PAP), central venous pressure (CVP), left ventricular pressure (LVP), and aortic pressure (AoP) during catheter pullback through the stent valve during dopamine infusion four hours after transarterial implantation. 


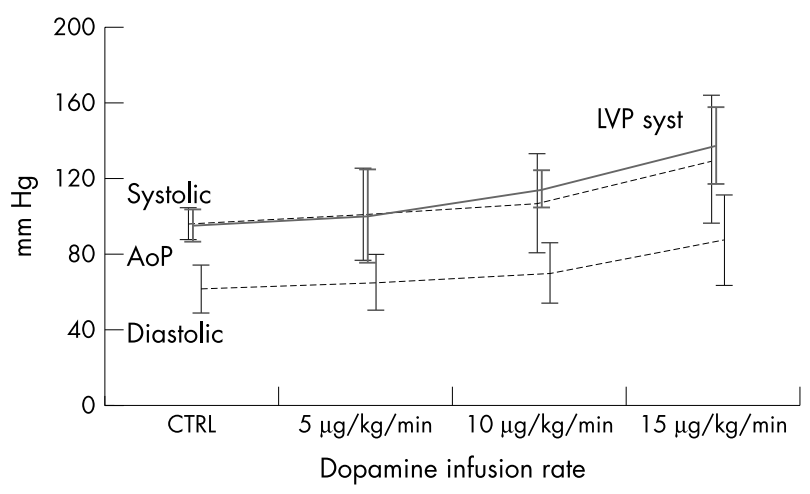

Figure 4 Aortic pressure (AoP, dotted lines) and systolic left ventricular pressure (LVP syst, bold line) during infusion of dopamine. Data are mean (SD). CTRL, baseline.

\section{Pharmacological stress testing}

Dopamine was infused at dosages of 5,10 , and $15 \mu \mathrm{g} / \mathrm{kg} / \mathrm{min}$ to create inotropic stress conditions. Each infusion was maintained for at least five minutes to achieve haemodynamic stability during recording. Aortic pressure, pulmonary artery pressure, pulmonary capillary wedge pressure, and central venous pressure were recorded continuously. We measured the pressure in the left ventricle by retrograde catheterisation with the pigtail catheter pulled back through the stent valve. $\mathrm{CO}$ was measured three times at each infusion rate of dopamine. Using the continuous wave Doppler TOE, we analysed the peak velocity in the implanted stent valve (AoVmax).

\section{RESULTS}

In four pigs $(67 \%)$ we replaced the aortic valve in the beating heart with no complication. Continuous haemodynamic registration did not show any relevant drop in blood pressure. The procedure failed in two pigs $(33 \%)$ because of dysfunction of the catheter device in one case and problems with correct positioning in the left ventricular outflow tract in the other, leading to acute aortic regurgitation and cardiac failure.

Pharmacological stress tests were performed in the remaining four pigs. During infusion of dopamine the CO increased from 3.8 to $8.8 \mathrm{l} / \mathrm{min}$. Under $15 \mu \mathrm{g} / \mathrm{kg} / \mathrm{min}$ dopamine the heart rate reached an average of 139 beats/min; in one pig we observed a heart rate of 180 beats/min. The arterial blood pressure and central venous pressure increased during dopamine infusion, whereas the pulmonary pressure remained unchanged. The pulmonary capillary wedge pressure deceased slightly during pharmacological stress testing. The left ventricular end diastolic pressure also decreased from 14.5 to $9.8 \mathrm{~mm} \mathrm{Hg}$ during infusion of dopamine.

The maximum peak to peak pressure gradient across the stent valve reached a maximum of $8 \mathrm{~mm} \mathrm{Hg}$ under dopamine infusion (figs 3 and 4). AoVmax measured by continuous wave Doppler TOE reached a maximum of $1.5 \mathrm{~m} / \mathrm{s}$. The calculated pressure gradient according to the modified Bernoulli formula $\left(4 \times\right.$ AoVmax $\left.^{2}\right)$ was $9.3 \mathrm{~mm} \mathrm{Hg}$. TOE measurements taken at the end of the experiment showed sufficient stent valve function in the left ventricular outlet tract (fig 5). Except in the pig that died of aortic regurgitation, continuous wave Doppler and colour Doppler analysis with TOE did not show regurgitation in the stent valve of any of the remaining four pigs.

Table 1 summarises the haemodynamic parameters measured before and after stent valve implantation and at the end of the experiment. Table 2 shows the haemodynamic reaction after dopamine infusion with increasing infusion speeds.

All pigs were killed six hours after transvascular aortic valve replacement. The chest was opened and the left ventricle and the ascending aorta were carefully inspected. The stent valve did not obstruct the coronary ostia. The original aortic valve was pushed against the aortic wall surrounding the stent valve. The valve prosthesis in the stent was completely unfolded in the orthotopic position. There

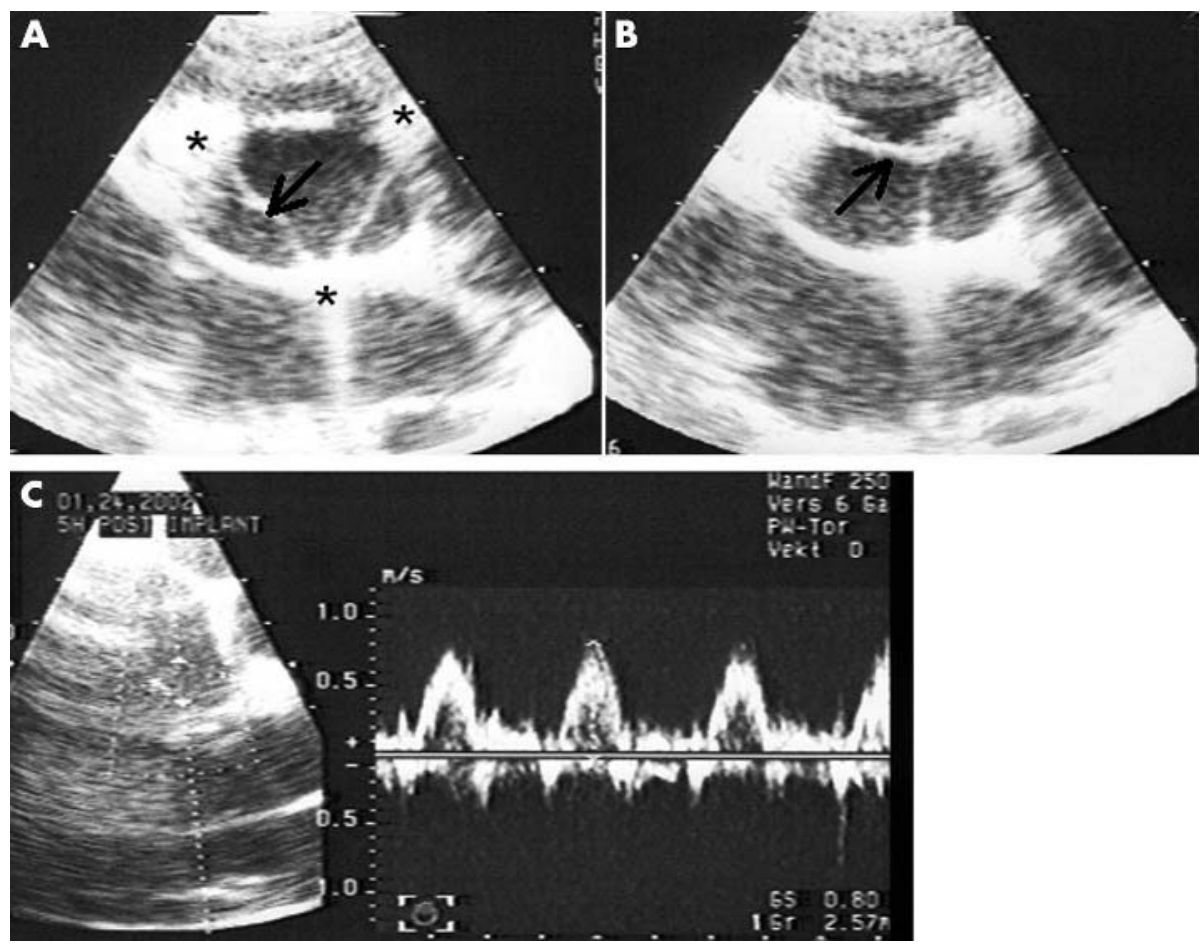

Figure 5 Transoesophageal echocardiography of the left ventricular outflow tract in a modified short axis view. Arrows show the cusps of the valve prosthesis in the (A) open and (B) closed valve prosthesis. The three ventricular arcs of the stent $\left(^{*}\right)$ can be seen. (C) Peak flow velocity in the aortic stent valve was measured with continuous wave Doppler. 
Table 1 Haemodynamic parameters before and after aortic stent valve implantation and at the end of the experiment

\begin{tabular}{llll}
\hline & $\begin{array}{l}\text { Before stent } \\
\text { implantation }\end{array}$ & $\begin{array}{l}\text { After stent } \\
\text { implantation }\end{array}$ & End of experiment \\
\hline HR (1/min) & $71.4(6.25)$ & $76.6(6.44)$ & $72.8(19.66)$ \\
PAP systolic (mm Hg) & $30.4(4.96)$ & $31.4(6.01)$ & $35.5(11.19)$ \\
PAP diastolic (mm Hg) & $15.0(3.35)$ & $14.8(3.56)$ & $16.8(6.30)$ \\
PAP mean (mm Hg) & $24.4(3.93)$ & $23.8(3.90)$ & $24.8(7.08)$ \\
PCW (mm Hg) & $16.2(6.52)$ & $14.5(6.98)$ & $12.0(6.04)$ \\
CVP (mm Hg) & $13.2(2.93)$ & $13.0(3.41)$ & $15.3(6.53)$ \\
LVP systolic (mm Hg) & $103.8(7.98)$ & $100.5(12.46)$ & $83.3(14.62)$ \\
LVP diastolic $(\mathrm{mm} \mathrm{Hg})$ & $1.2(1.60)$ & $1.0(1.73)$ & $0.5(0.87)$ \\
LVP end diastolic $(\mathrm{mm} \mathrm{Hg})$ & $14.6(6.62)$ & $14.5(6.98)$ & $12.3(5.85)$ \\
AoP systolic $(\mathrm{mm} \mathrm{Hg})$ & $103.4(8.31)$ & $98.0(12.21)$ & $83.3(15.83)$ \\
AoP diastolic $(\mathrm{mm} \mathrm{Hg})$ & $71.4(10.44)$ & $65.0(11.15)$ & $50.5(6.38)$ \\
AoP mean $(\mathrm{mm} \mathrm{Hg})$ & $88.5(7.31)$ & $84.6(9.51)$ & $65.6(11.76)$ \\
CO (I/min) & $3.8(0.73)$ & $3.7(0.87)$ & $3.9(1.39)$ \\
AoVmax $(\mathrm{m} / \mathrm{s})$ & $0.7(0.06)$ & $0.8(0.11)$ & $0.6(0.14)$ \\
\hline
\end{tabular}

Presented parameters are mean (SD) values of four pigs.

AoP, aortic pressure; AoVmax, peak velocity in the aortic valve/implanted stent valve measured by continuous wave Doppler; CO, cardiac output; CVP, central venous pressure; HR, heart rate; LVP, left ventricular pressure; PAP, pulmonary artery pressure; PCW, pulmonary capillary wedge pressure.

were no signs of damage or malfunction of the implanted stent valve, nor of damage of the aortic vessel wall. The mitral valve was not affected. The explanted pig hearts weighed 390 (27.4) g.

\section{DISCUSSION}

Surgical replacement of diseased aortic valves has been available for half a century. ${ }^{12}$ Progress in the surgical technique, and the quality of the implanted valves and of the post-procedural medical treatment, has lowered the surgical risk for most patients. ${ }^{13}$ Even patients aged 80 years and older benefit from an aortic valve replacement with an acceptable risk if they do not have relevant co-morbidity. ${ }^{14}$ However, owing to increasing longevity we are faced with a rising number of high risk elderly patients with various comorbidities who need aortic valve replacement. ${ }^{15}{ }^{16}$

Balloon valvoplasty was performed in patients with aortic valve stenosis with satisfying short term results. ${ }^{17}$ But the high restenosis rate within a few months discouraged cardiologists from performing this procedure as an alternative to surgical valve replacement. ${ }^{18}$ This treatment dilemma in elderly high risk patients demands a minimally invasive technique of aortic valve replacement without the need for cardiopulmonary bypass and circulatory arrest.

We showed in the presented study the feasibility of transarterial aortic valve replacement in the beating heart.
The success rate of percutaneous implantation of a self expanding stent valve was $67 \%$ in our study with no balloon inflations in the ascending aorta. For the first time, this technology was successfully tested in an experimental model under haemodynamic stress conditions. The two unsuccessful attempts can be explained by false positioning in one case and by technical limitations of the implantation catheter device.

However, in the other experiments the design of the stent allowed exact implantation of the stent valve in the left ventricular outlet tract with physiological function of the valve prosthesis. Its continuous expansion force in combination with barbed hooks guaranteed secure anchoring of the implant in the ascending aorta. During dopamine infusion CO increased to $240 \%$ relative to the baseline value. The systolic aortic pressure increased from 98 to $130 \mathrm{~mm} \mathrm{Hg}$, and the diastolic aortic pressure rose from 65 to $87 \mathrm{~mm} \mathrm{Hg}$ during the highest dose of dopamine. Under a maximum heart rate of nearly 140 beats/min no relevant dysfunction of the stent valve occurred. Left ventricular end diastolic pressure and pulmonary capillary wedge pressure decreased slightly during the stress test. Left ventricular function was not impaired by the stent valve.

At rest the peak pressure gradient in the stent valve prosthesis was $2.5 \mathrm{~mm} \mathrm{Hg}$. A peak pressure gradient of $8 \mathrm{~mm} \mathrm{Hg}$ measured by catheter pullback through the stent

Table 2 Haemodynamic parameters during stress test with intravenous infusion of dopamine

\begin{tabular}{|c|c|c|c|}
\hline & \multicolumn{3}{|c|}{ Rate of dopamine infusion } \\
\hline & $5 \mu \mathrm{g} / \mathrm{kg} / \mathrm{min}$ & $10 \mu \mathrm{g} / \mathrm{kg} / \mathrm{min}$ & $15 \mu \mathrm{g} / \mathrm{kg} / \mathrm{min}$ \\
\hline $\mathrm{HR}(1 / \mathrm{min})$ & $94.5(10.80)$ & $100.0(13.55)$ & $139.2(33.48)$ \\
\hline PAP systolic (mm Hg) & $34.3(4.82)$ & $35.0(4.86)$ & $35.7(5.96)$ \\
\hline PAP diastolic $(\mathrm{mm} \mathrm{Hg})$ & $17.5(4.54)$ & $17.3(4.27)$ & $19.5(5.41)$ \\
\hline PAP mean $(\mathrm{mm} \mathrm{Hg})$ & $26.3(2.87)$ & $25.5(3.99)$ & $27.0(4.12)$ \\
\hline PCW $(\mathrm{mm} \mathrm{Hg})$ & $14.5(7.25)$ & $11.5(6.92)$ & $10.0(5.69)$ \\
\hline CVP $(\mathrm{mm} \mathrm{Hg})$ & $16.0(5.23)$ & $18.2(4.74)$ & $20.8(5.27)$ \\
\hline LVP systolic (mm Hg) & $101.0(24.54)$ & $114.8(10.09)$ & 137.7 (19.96) \\
\hline LVP diastolic (mm Hg) & $1.3(4.11)$ & $0.2(3.29)$ & $-0.3(3.86)$ \\
\hline LVP end diastolic (mm Hg) & $13.8(7.71)$ & $11.7(6.77)$ & $9.8(5.81)$ \\
\hline AoP systolic $(\mathrm{mm} \mathrm{Hg})$ & $101.0(24.54)$ & $106.5(26.39)$ & 129.8 (34.28) \\
\hline AoP diastolic $(\mathrm{mm} \mathrm{Hg})$ & $64.3(14.88)$ & $69.3(15.98)$ & 86.7 (23.72) \\
\hline AoP mean $(\mathrm{mm} \mathrm{Hg})$ & $78.6(18.78)$ & $86.4(20.30)$ & $104.8(27.64)$ \\
\hline CO $(1 / \mathrm{min})$ & $5.5(1.39)$ & $7.0(1.33)$ & $8.8(0.63)$ \\
\hline AoVmax $(\mathrm{m} / \mathrm{s})$ & $0.9(0.14)$ & $1.2(0.10)$ & $1.5(0.16)$ \\
\hline
\end{tabular}


valve excluded a relevant outflow obstruction. Mean systolic transprosthetic pressure gradients reported by other groups testing a balloon expandable stent were 5-6 mm Hg under resting conditions. ${ }^{71}$ The pressure gradient in our study was $2.5 \mathrm{~mm} \mathrm{Hg}$ at rest and increased to $8 \mathrm{~mm} \mathrm{Hg}$ during pharmacological stress. However, no other group performed stress testing in their animal experiments.

However, we implanted the stent valve in healthy ascending aortas of pigs. Whether the stent may additionally obstruct the left ventricular outlet tract in heavily calcified aortic stenosis has to be addressed in the future. A preceding balloon valvoplasty will be essential in these patients before implantation of the self expanding stent valve, since the expansion force of the stent will not be able to dilate the stenotic valve.

Cribier and colleagues ${ }^{10}$ implanted a percutaneous stent valve in the aortic position in a human a few months ago. In this last resort case, they used a balloon expandable stent, which was inserted through a transseptal approach after successful valvoplasty. With this technology of balloon expandable stent valves, patients are at risk of paravalvar regurgitation, which may slowly increase due to chronic mechanical stress on the stent, and retraction of the surrounding tissue. Follow up echocardiography of the aortic valve area showed good intermediate results. These data suggested that implantation of a stent valve after balloon valvoplasty may improve long term results compared with standalone valvoplasty of stenotic aortic valves. However, patients with an aortic stenosis with cardiogenic shock are a very high risk group. ${ }^{20}$ The self expanding force of the above described stent would help to improve the long term result of an emergency valvoplasty in these desperate cases.

The two technologies of stent valve implantation (balloon expandable versus self expanding) have different advantages. The transseptal approach of the balloon expandable stent is also feasible in patients with severe peripheral atherosclerosis. But transseptal access to the aortic valve is prone to more complications. The retrograde approach with a self expanding stent has advantages in patients with aortic regurgitation. While anchoring a balloon expandable stent in a non-calcified regurgitant aortic valve is nearly impossible, the self expanding stent can adapt its mechanical force to the dilated aortic ring. The retrograde approach allows implantation of longer stents. While patients with a less calcified aortic ring are susceptible to dislocation after implantation of a balloon expandable short stent, the longer self expanding stent safely anchors itself in the aortic ring and in the ascending aorta. Patients with acute aortic regurgitation caused by endocarditis are another high risk group for open surgical valve replacement. ${ }^{21}$ With a balloon inflated stent valve it would be necessary to inflate the balloon in the left ventricular outlet tract for half a minute. The technology of a self expanding stent valve for emergency aortic valve replacement would be favourable in those cases as well. On the other hand, it is unclear whether the self expanding stent would lead to a higher incidence of late aortic rupture as described for elderly women after surgical repair of aortic regurgitation..$^{22}$ Long term animal experiments should answer this question.

However, the majority of patients with severe aortic valve disease benefit from open surgical valve replacement. The percutaneous stent valve will be a complementary tool for treating patients with a high risk for open heart surgery due to age and co-morbidities. Besides technical improvements of the implantation device, especially further reduction of its diameter, long term animal experiments are necessary before this technology of percutaneous stent valve can be used for patients other than as a last resort.

\section{Conclusion}

This study proves the feasibility of percutaneous aortic valve replacement in the beating heart and shows the efficiency of the device under pharmacological stress. With further diameter reduction of the implantation catheter and long term animal experiments this concept may become a feasible option for treating patients with relevant aortic valve disease, but at high operative risk in the near future.

\section{ACKNOWLEDGMENTS}

This study was supported by funding from the German Ministry of Research and Technology (BMBF) and the German Aerospace Centre (DLR) \# $01 E Z 003$.

\section{Authors' affiliations}

M Ferrari, H R Figulla, M Schlosser, I Tenner, G S Werner, Clinic of

Internal Medicine, Friedrich-Schiller University, Jena, Germany

I Frerichs, G Hellige, Cardiac Centre, Georg-August University,

Goettingen, Germany

C Damm, V Guyenot, Fraunhofer Institute of Applied Optics and Micromechanics, Jena, Germany

\section{REFERENCES}

1 Kelly TA, Rothbart RM, Cooper CM, et al. Comparison of outcome of asymptomatic to symptomatic patients older than 20 years of age with valvular aortic stenosis. Am J Cardiol 1988;61:123-30.

2 Lindroos M, Kupari M, Heikkila J, et al. Prevalence of aortic valve abnormalities in the elderly: an echocardiographic study of a random population sample. J Am Coll Cardiol 1993;21: 1220-5.

3 Roques F, Nashef SA, Michel P, et al. Risk factors and outcome in European cardiac surgery: analysis of the EuroSCORE multinational database of 19030 patients. Eur J Cardiothorac Surg 1999;15:816-22.

4 Edwards MB, Taylor KM. Outcomes in nonagenarians after heart valve replacement operation. Ann Thorac Surg 2003;75:830-4.

5 Andersen HR, Knudsen LL, Hasenkam JM. Transluminal implantation of artificial heart valves: description of a new expandable aortic valve and initial results with implantation by catheter technique in closed chest pigs. Eur Heart $J$ 1992;13:704-8.

6 Sochman J, Peregrin JH, Pavcnik D, et al. Percutaneous transcatheter aortic disc valve prosthesis implantation: a feasibility study. Cardiovasc Intervent Radiol 2000;23:384-8.

7 Boudjemline $Y$, Bonhoeffer P. Steps toward percutaneous aortic valve replacement. Circulation 2002;105:775-8.

8 Paniagua D, Induni E, Ortiz C, et al. Images in cardiovascular medicine. Percutaneous heart valve in the chronic in vitro testing model. Circulation 2002; 106:e51-2

9 Bonhoeffer $\mathbf{P}$, Boudjemline $Y$, Saliba Z, et al. Percutaneous replacement of pulmonary valve in a right-ventricle to pulmonary-artery prosthetic conduit with valve dysfunction. Lancet 2000;356:1403-5.

10 Cribier A, Eltchaninoff H, Bash A, et al. Percutaneous transcatheter implantation of an aortic valve prosthesis for calcific aortic stenosis: first human case description. Circulation 2002;106:3006-8.

11 Council of Europe. European convention for the protection of vertebrate animals used for experimental and other scientific purposes. European Treaty Series, no 123. Strasbourg: Council of Europe, 1986.

12 Hufnagel CA, Harvey WP. The surgical correction of aortic regurgitation preliminary report. Bull Georgetown Univ Med Cent 1953;6:60-1.

13 Roques F, Nashef SA, Michel P. Regional differences in surgical heart valve disease in Europe: comparison between northern and southern subsets of the EuroSCORE database. J Heart Valve Dis 2003;12:1-6.

14 Gehlot A, Mullany CJ, Ilstrup D, et al. Aortic valve replacement in patients aged eighty years and older: early and long-term results. J Thorac Cardiovasc Surg 1996;111:1026-36.

15 Freeman WK, Schaff HV, O'Brien PC, et al. Cardiac surgery in the octogenarian: perioperative outcome and clinical follow-up. J Am Coll Cardiol $1991 ; 18: 29-35$.

16 Iskandrian AS, Segal BL. Should cardiac surgery be performed in octogenarians? J Am Coll Cardiol 1991;18:36-7.

17 Cribier A, Savin T, Saoudi N, et al. Percutaneous transluminal valvuloplasty of acquired aortic stenosis in elderly patients: an alternative to valve replacement? Lancet 1986;i:63-7.

18 Feldman T, Glagov S, Carroll JD. Restenosis following successful balloon valvuloplasty: bone formation in aortic valve leaflets. Cathet Cardiovasc Diagn 1993;29:1-7.

19 Lutter G, Kuklinski D, Berg G, et al. Percutaneous aortic valve replacement: an experimental study. I. Studies on implantation. J Thorac Cardiovasc Surg 2002;123:768-76.

20 Cribier A, Remadi F, Koning R, et al. Emergency balloon valvuloplasty as initial treatment of patients with aortic stenosis and cardiogenic shock. N Engl J Med 1992;326:646. 
21 Pilegaard $\mathrm{HK}$, Lund $\mathrm{O}$, Nielsen $\mathrm{TT}$, et al. Early and late prognosis after valve replacement in aortic regurgitation: preoperative risk stratification and reasons for a more aggressive surgical approach. Thorac Cardiovasc Surg 1989;37:231-7.
22 McDonald ML, Smedira NG, Blackstone EH, et al. Reduced survival in women after valve surgery for aortic regurgitation: effect of aortic enlargement and late aortic rupture. J Thorac Cardiovasc Surg 2000:119:1205-12.

\section{IMAGES IN CARDIOLOGY}

\section{Acute massive pulmonary thromboembolism simulating anterolateral myocardial ischaemia}

A 71 year old man with a history of hypercholesterolaemia and smoking was admitted to the coronary care unit because of typical chest pain and dyspnoea at rest. One month before admission he underwent hip prosthesis replacement and had been treated with low molecular weight heparin (prophylactic dose), which was discontinued three days before the onset of symptoms. An ECG showed sinus tachycardia with right bundle branch block, ST segment depression, and deep negative $\mathrm{T}$ waves in leads Vl-V5, suggesting severe anterolateral myocardial ischaemia (panel A). Troponin I was elevated. The patient, initially diagnosed as acute coronary syndrome, was treated with aspirin, low molecular weight heparin, and platelet glycoprotein IIb/IIIa inhibitors. Two hours later, emergency cardiac catheterisation was performed for haemodynamic instability (systolic arterial pressure of $70 \mathrm{~mm} \mathrm{Hg}$ ). Cardiac catheterisation showed normal coronary angiograms and pulmonary systolic pressure at the systemic level (panel B). Pulmonary angiography revealed complete thromboembolic occlusion by a large and mobile thrombus in the right pulmonary artery (panel C). The patient

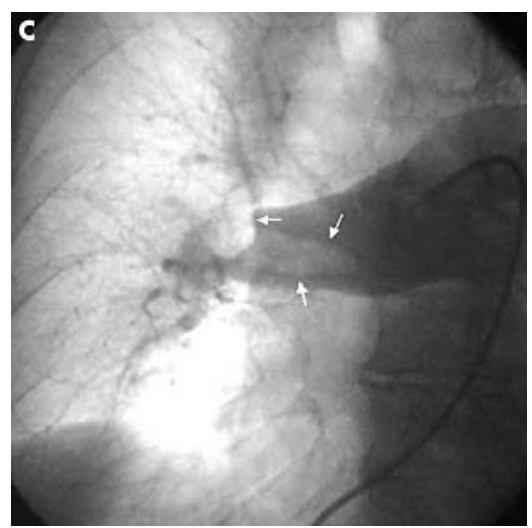

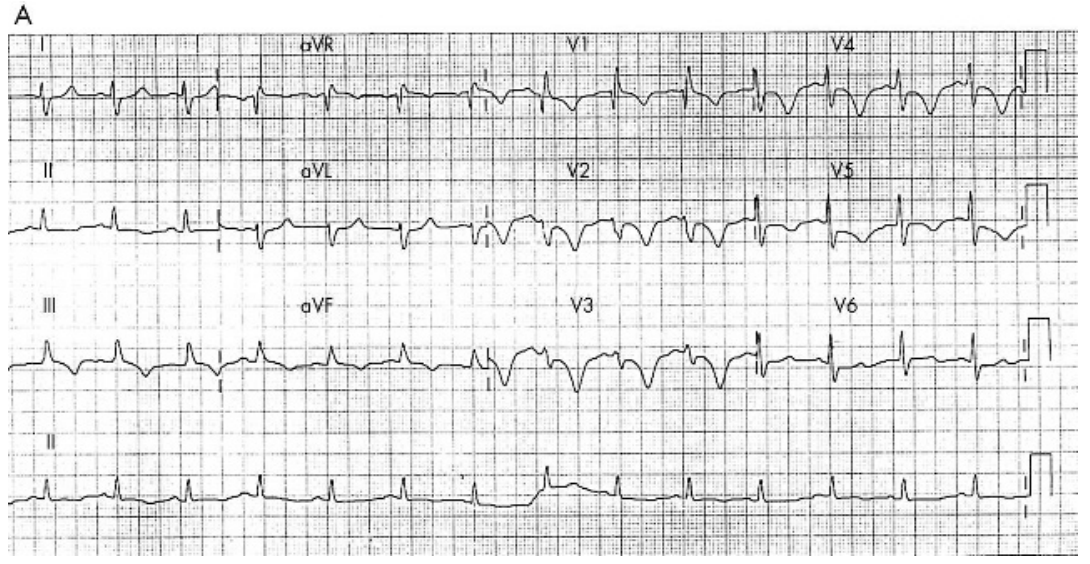

B

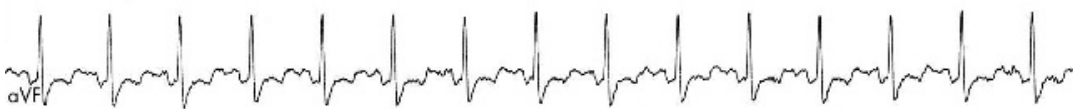

200

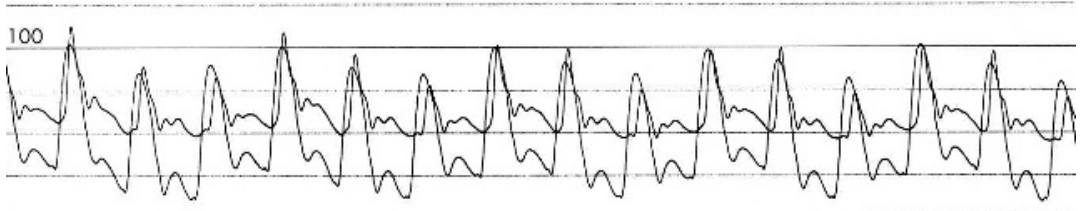

developed electromechanical dissociation and died during catheterisation.

This case illustrates the importance of clinical suspicion for the early diagnosis and treatment of pulmonary embolism.
A Sanchez-Recalde G Galeote R Sanchez-Aquino asanchezr.hulp@salud.madrid.org 